\title{
Contact De-electrification of Electrostatically Charged Polymers
}

\section{Citation}

Soh, Siowling, Sen Wai Kwok, Helena Liu, and George M. Whitesides. 2012. Contact DeElectrification of Electrostatically Charged Polymers. Journal of the American Chemical Society 134, no. 49: 20151-20159.

\section{Published Version}

doi:10.1021/ja309268n

\section{Permanent link}

http://nrs.harvard.edu/urn-3:HUL.InstRepos:11933752

\section{Terms of Use}

This article was downloaded from Harvard University's DASH repository, and is made available under the terms and conditions applicable to Open Access Policy Articles, as set forth at http:// nrs.harvard.edu/urn-3:HUL.InstRepos:dash.current.terms-of-use\#OAP

\section{Share Your Story}

The Harvard community has made this article openly available.

Please share how this access benefits you. Submit a story.

Accessibility 


\title{
Contact de-Electrification of Electrostatically Charged Polymers
}

\author{
Siowling Soh, Sen Wai Kwok, Helena Liu, and George M. Whitesides*
}

Department of Chemistry and Chemical Biology, Harvard University, 12 Oxford St. Cambridge, MA 02138, U.S.A.

* To whom correspondence may be addressed: gwhitesides@gmwgroup.harvard.edu 


\begin{abstract}
:
The contact electrification of insulating organic polymers is still incompletely understood, in part because multiple fundamental mechanisms may contribute to the movement of charge. This study describes a mechanism previously unreported in the context of contact electrification: that is, "contact de-electrification", a process in which polymers charged to the same polarity discharge on contact. Both positively charged polymeric beads, e.g., polyamide 6/6 (Nylon) and polyoxymethylene (Delrin), and negatively charged polymeric beads, e.g., polytetrafluoroethylene (Teflon) and polyamide-imide (Torlon), discharge when the like-charged beads are brought into contact. The beads (both with charges of $\sim \pm 20 \mu \mathrm{C} / \mathrm{m}^{2}$, or $\sim 100$ charges $/ \mu \mathrm{m}^{2}$ ) discharge on contact regardless of whether they are made of the same material, or of different materials. Discharge is rapid: discharge of flat slabs of like-charged Nylon and Teflon pieces is completed on a single contact $(\sim 3 \mathrm{sec})$. The charge lost from the polymers during contact de-electrification transfers onto molecules of gas in the atmosphere. When likecharged polymers are brought into contact, the increase in electric field at the point of contact exceeds the dielectric breakdown strength of the atmosphere and ionizes molecules of the gas; this ionization thus leads to discharge of the polymers. The detection (using a Faraday Cup) of charges transferred to the Cup by the ionized gas is compatible with the mechanism. Contact deelectrification occurs for different polymers and in atmospheres with different values of dielectric breakdown strength (helium, argon, oxygen, carbon dioxide, nitrogen, and sulfur hexafluoride): the mechanism thus appears to be general.
\end{abstract}




\section{INTRODUCTION}

In contact electrification, a difference in electrical potential develops when two materials are brought into contact (either frictional, or, ostensibly, static) and then separated. Although contact electrification has been studied for more than 2500 years, the underlying physical mechanisms that govern the separation of charge remain incompletely defined. ${ }^{1-3}$ Understanding the mechanisms related to electrification involving contact among multiple bodies is important in controlling, for example, the flow of granular matter, the processing of pharmaceutical powders, electrophotography (e.g. photocopying and printing), the movement of small particles (e.g. sand or fragments of grain) in dust storms and dust clouds (with the possibility of dust explosions), and the movement of air, water droplets and ice particles in storms (and the generation of lightning). ${ }^{4}$

It is probable that contact electrification involves multiple physical mechanisms depending on materials and circumstance. These mechanisms include transfer of ions, electrons, or small particles of charged matter, shear in fluids near interfaces, fracture of electrically polarized solids

and others. ${ }^{1,5,6}$ The combination of multiple mechanisms operating concurrently may underlie the variations in the ordering of materials in the triboelectric series (an empirically organized list of materials based on their tendency to charge either positively or negatively), in which the sequence differs slightly among different experimental studies. ${ }^{7}$ In particular, one example is provided by the observation that some sets of materials (for example, glass, zinc, silk, filter paper, and cotton) form cyclic triboelectric series — an indication that there may be more than one physical mechanism responsible for contact electrification. ${ }^{1,5}$ Another example involves the reversal of charge sometimes observed for two different materials when they are brought into contact over an interval of time. ${ }^{8}$ For example, a bead (polytetrafluoroethylene) charged initially 
negatively when in contact with a dish (polystyrene) could become positively charged after contact with the same dish for longer times. This reversal in polarity suggests the simultaneous operation of different mechanisms, with different rate constants. It is necessary to identify the most important mechanisms involved in contact electrification, in order to understand and control the tribocharging of granular particles.

Contact electrification is intrinsically a dynamic and dissipative process: that is, mechanical work is required to generate separated charge. In all systems, the amount of charge separated at steady-state ultimately reaches a limit. A process that reaches a steady-state suggests the possibility of competing processes that involve both charging and discharging. This paper describes what we will call "contact de-electrification", a surprising (at least initially) phenomenon that has not previously been described in the context of tribocharging: contact of two already-charged particles with the same polarity that results in their discharge (Figure 1).

In a typical process resulting in contact electrification, contact between two different materials results in the separation of charge; one material charges positively, while the other material charges negatively. Overall, this type of process seems to conserve charge between the materials (Figure 1). Interestingly, particles of the same material can sometimes also separate charge upon contact; one particle will charge positively, while the second particle of the same material will charge negatively, again with overall conservation of charge. ${ }^{9,10}$ 

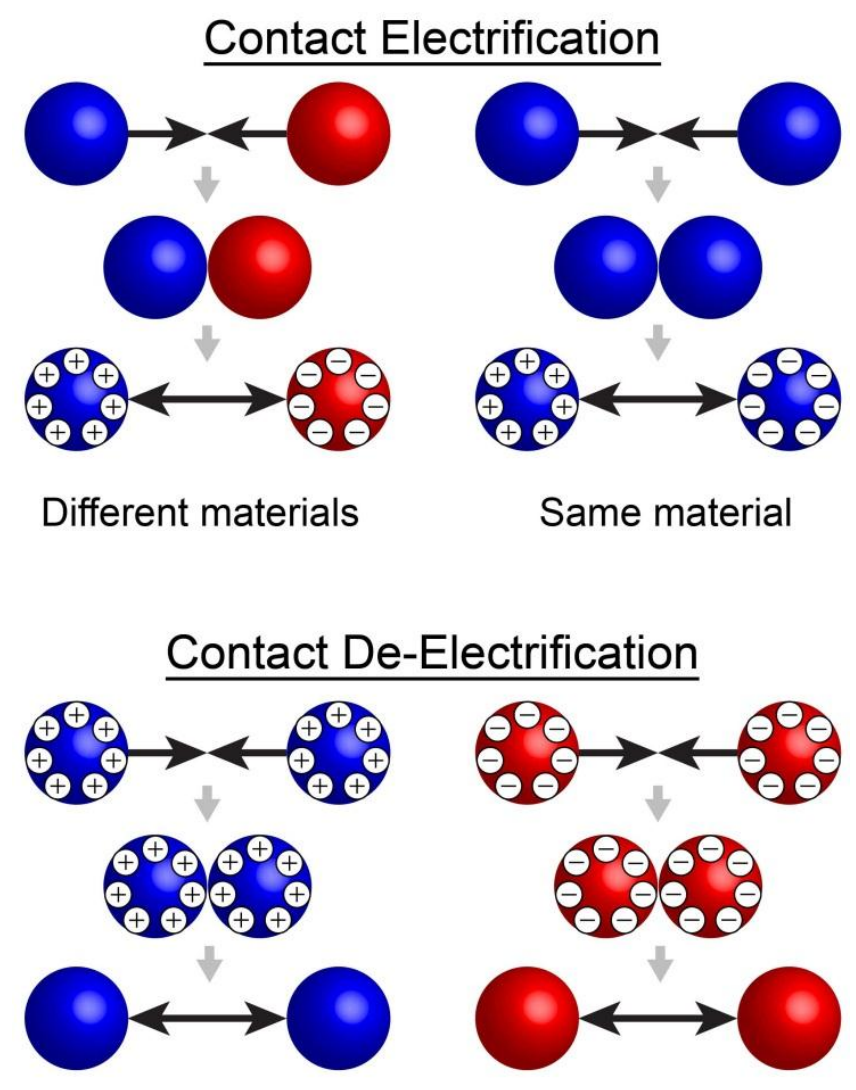

Figure 1. Contact electrification and contact de-electrification. For contact electrification, when two uncharged objects of different materials are brought into contact and then separated, one of them charges positively, while the other charges negatively (top left scheme). The overall charge on the objects is conserved. The same effect has also been demonstrated for two objects of the same material (top right scheme). In this study, we describe a phenomenon in which the contact between two initially charged objects results in discharge - a phenomenon, which we refer to as "contact de-electrification" (bottom scheme). Both initially positively and negatively charged objects experience the same discharge phenomenon after contact. 
Contact Electrification by Electron Transfer. For metal-metal contacts, electrons are the dominant mobile charged species ${ }^{11}$ as demonstrated from experiments in which the potential difference developed between the surfaces of the metals after contact is linearly proportional to the difference in their work functions. For metal-insulator contact, similar trends with the work function of the metal have been claimed, and interpreted to suggest that the charge carrier is also an electron; this result is, however, not universal for all types of polymers, ${ }^{11,12}$ and is also open to other interpretations. There are also suggestions that electrons (or, peculiarly, species called "cryptoelectrons") are the charge carrier for insulator-insulator contacts; ${ }^{13}$ cryptoelectrons are now generally regarded with skepticism. ${ }^{14-16}$

Contact Electrification by Ion Transfer. Although separation of charges by movement of electrons is important for metals, it is probably not important in charge separation involving only insulators. Instead, in the case of insulating surfaces covered with covalently bound ionic functional groups and mobile counterions, it is clear that the charge carriers are the mobile counterions. ${ }^{1,17-19}$ It is possible, although not proved, that this ion transfer mechanism is general for all types of insulator-insulator contacts. ${ }^{1,5}$

Adsorption of Hydroxide Ions from Thin Water Film. Non-ionic insulators (e.g., polytetrafluoroethylene and polystyrene) also develop charge readily by triboelectrification. The mechanism is less clear than for ionic solids, and may require water. Practically all solid surfaces, including hydrophobic surfaces, adsorb water from the atmosphere. Experimentally, when two solid surfaces are brought into contact, the surface with a higher affinity for hydroxide ions (or, more generally, negatively charged ions) will adsorb more of the hydroxide ions from the film of adsorbed water than that with the lower affinity, and gain a net negative charge when the surfaces are separated. ${ }^{1}$ This proposed mechanism is supported ${ }^{1}$ by an approximately linear 
relationship between the zeta potential and the amount of charge separated after tribocharging different types of polymer.

Contact Electrification by Material Transfer. Charge transfer can also occur when quantities of charged microscopic materials detach from their original surface and attach to the other contacting surface. Recent results show that material transfer appears to be significant in some types of contact electrification, ${ }^{3,8,14}$ but whether this transfer causes charge separation or simply correlates with it is difficult to establish.

Mechanisms of Loss of Charge. Mechanisms for loss of charge are not well-studied and understood, especially for insulators. Highly charged solid surfaces can lose charge when the electric field on the surface exceeds the dielectric breakdown strength of the surrounding atmosphere. Examples include field ionization, corona discharge, electrical arcing, partial discharge (one process responsible for electrical failure of insulating materials), and spontaneous discharge during tribocharging. ${ }^{20,21}$ Charge can also leak from a charged solid to other grounded solid surfaces via a layer of adsorbed water on the surface of the solid. ${ }^{1}$

This paper explores the mechanism of contact de-electrification for charged, insulating polymeric beads. In these experiments, we first charge beads to the same polarity and then bring the charged beads into contact; this contact results in discharge of the beads. Since charge must be conserved, the charges that disappear from contacting the like-charged particles must appear elsewhere. Where? We propose, in the systems studied, that charged polymeric beads discharge by transfer of charges from the beads to the molecules of gas in the atmosphere. 


\section{EXPERIMENTAL DESIGN}

Choice of Materials. The polymeric beads used in the experiments were Nylon (polyamide 6/6), Delrin (polyoxymethylene), Teflon (polytetrafluoroethylene), and Torlon (polyamide-imide) beads. The reason for using these polymers was because Nylon and Delrin charged highly positively $\left(\sim 20 \mu \mathrm{C} / \mathrm{m}^{2}\right)$ while Teflon and Torlon charged highly negatively $(\sim-20$ $\mu \mathrm{C} / \mathrm{m}^{2}$ ) in our experiments, and in accordance with the triboelectric series. ${ }^{7}$ We used polybutylene terephthalate tweezers for all the physical manipulations of the beads since the charges on the beads did not change appreciably after handling (more details later). To minimize the influence of humidity, all experiments were conducted inside a glove bag under an atmosphere of nitrogen (unless otherwise specified) at $\sim 1-2 \%$ relative humidity.

We measured the charges of the beads using a Faraday Cup — a measuring device that consists of two layers of metal separated by insulating foam, with the inner layer acting as an electrode and the outer layer grounded - connected to an electrometer. ${ }^{22}$ The electrometer measured the total charge of any objects placed in the Faraday Cup by recording the equal, but opposite, amount of charge, $Q$, induced on the electrode of the Faraday Cup. This amount of charge was stored in an accurately known capacitor, with capacitance, $C$, in the electrometer. The electrometer then measured the potential difference, $V$, across the capacitor and translated $V$ into $Q$ by the equation $Q=C V$.

Procedure for Charging Beads. To examine contact de-electrification, we first charged the beads by contact electrification through agitating two types of polymeric beads - one type of polymeric beads charged positively, while the other type charged negatively on contact loosely packed on a dish with a motor (Figure 2). The advantages of this method, as opposed to 
contacting surfaces of the polymers manually, are: i) the polymeric beads charge consistently to approximately the same extent on agitation (Figure 3); ii) charging using motor-driven agitation is self-regulating and independent of the operator; iii) the steady-state charge is high (in our case, $\sim \pm 20 \mu \mathrm{C} / \mathrm{m}^{2}$, or $\sim 100$ charges $/ \mu \mathrm{m}^{2}$ or 1 charge $/ 10^{4} \mathrm{~nm}^{2}$ ).

An alternative way of charging the beads is by using an antistatic "Zerostat" gun. Although both triboelectrification and deposition of externally generated charge have the same electrostatic outcome, they do not necessarily have the same mechanism. It is our intention to show that contact de-electrification occurs for materials that are charged by contact electrification (not, e.g., by deposition of charges on the surface). Another disadvantage of charging with the "Zerostat" gun is that it did not charge the beads to the same level as tribocharging: the amount of charge deposited by the "Zerostat" gun was only $~ 10-20 \%$ of that obtained using the tribocharging method we describe.

Procedure for Discharging Beads. After charging the beads, the process of discharging involved bringing two like-charged beads into contact. One way of bringing the beads into contact was to hold the two beads using two pairs of tweezers, with one pair of tweezers for each bead. This method (and other methods similar to this procedure), however, involved constant physical interaction between the charged beads and other solid substrates (e.g., pairs of tweezers). This interaction could, in principle, allow transfer of charge between the bead and the solid substrate, and would complicate the discussion of contact de-electrification. In order to avoid charge transfer from the beads to other solid substrates, we devised a method to hold the beads so that they could be brought into contact with minimal interaction between the beads and other solid substrates. 
This method consisted of using a substrate (polymethyl-methacrylate, PMMA) with five pockets arranged in a pentagon for inserting five charged beads ("side beads") into the pockets (Figure 2 and Figure $\mathrm{S} 2$ in the SI). These five charged beads then served as a bottom platform: we placed one of the two beads to be brought into contact ("center bead") in between and on top of the five beads. Importantly, this center bead was in contact with only like-charged beads and not with any other solid substrate. We immobilized the other bead to be brought into contact ("top bead") on an adhesive surface (a solid polystyrene surface with one of its surfaces covered with double-sided tape); by immobilizing the top bead, there was minimal interaction between the top bead and the adhesive solid surface ("top substrate").

Discharge occurred by bringing the top bead into contact with the center bead. Specifically, we brought the top substrate (with the top bead on the substrate) toward the other charged beads and pressed the top bead against the center bead. We then rubbed (with a force that weighed on the order of $10^{2} \mathrm{~g}$ on a balance for $\sim 10-20 \mathrm{~s}$ ) the surfaces available to the top and center beads manually by moving the top substrate, while keeping the top bead constantly pressed against the center bead. The bottom half of the center bead, however, was not accessible to the top bead. The center bead was, therefore, rotated through an angle of $180^{\circ}$ (Figure 2, bottom left scheme) using a pair of tweezers, so that the bottom, unexposed surface of the center bead became available for discharge. To ensure that the entire surface of the center bead came into contact with the top bead, the sequence of rotating and rubbing the beads was repeated in four different directions (Figure 2, bottom right). We also performed the whole procedure again, so that the center bead was rotated for a total of eight times, with the process lasting $\sim 3$ mins. See SI, Section 1 for more details on the experimental procedure. 

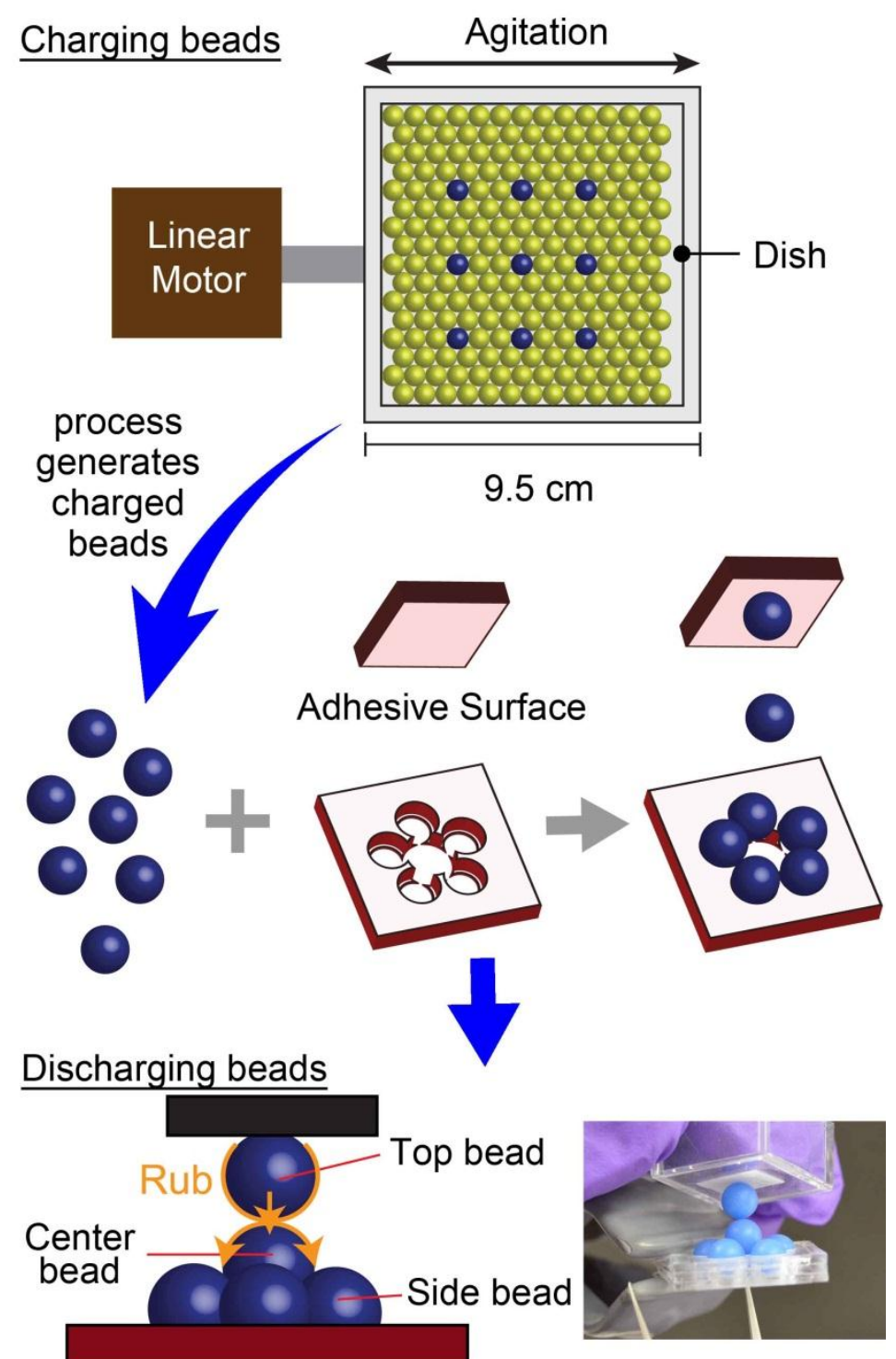

$\underline{\text { Rotating center bead }}$
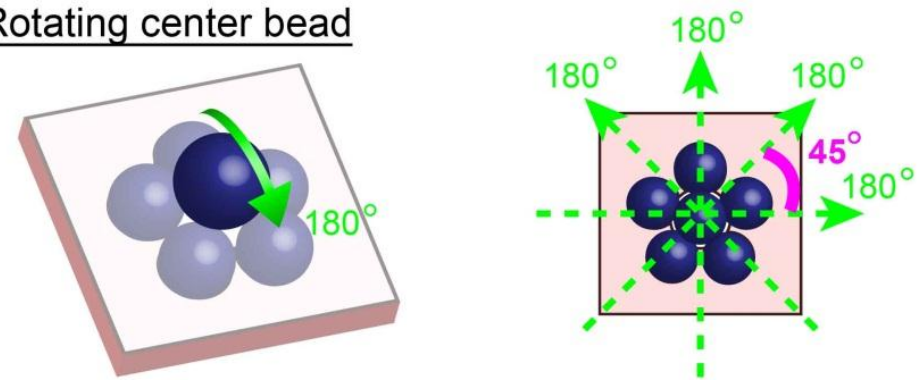
Figure 2. Experimental setup for demonstrating contact de-electrification. The top scheme illustrates the experimental setup for charging nine beads of a desired polymeric material (blue beads) against an excess number of beads of a different material that charged oppositely (yellow beads) in a dish. Beads were charged by contact electrification when they rotated and collided with one another on the dish agitated by a motor. After charging, seven beads (blue) were transferred to a platform: five beads (side beads) were placed on a bottom substrate, a sixth bead (center bead) was placed in between and on top of the five beads and a seventh bead (top bead) was immobilized on an adhesive surface. Importantly, this arrangement of the beads ensured that the center bead was only in physical contact with the side and top beads and not with any other solid substrates. Contact de-electrification occurred when the top bead was rubbed against the center bead. We rotated the center bead by $180^{\circ}$ (bottom left scheme) so that the bottom half of the bead could be brought into contact with the top bead. We also rotated the center bead along four different directions (bottom right scheme) and rubbed the top bead against the center bead after each rotation. 


\section{RESULTS AND DISCUSSION}

Materials. We used polymeric beads (Nylon, Teflon, Delrin and Torlon), 1/4-inch in diameter, in our experiments. The colorless Nylon beads were dyed blue (disperse blue dye) so that they were visually distinguishable from other colorless beads. Experiments performed on undyed beads showed that dyed and undyed beads behaved similarly (see SI, Section 2). A square aluminum dish (inner length $8.8 \mathrm{~cm}$ ) was used as a container to hold the beads. The type of dish did not matter: beads of different materials charged similarly on an aluminum (conductor) dish, a grounded aluminum dish, and a poly (methyl methacrylate) (insulator) dish. ${ }^{23}$ This observation further indicates that the charges on the beads are not electrons; otherwise the electrons on the beads would have dissipated into ground in a grounded aluminum dish. Before the experiments, we rinsed the beads and dish with deionized water and ethanol, and dried them in a stream of nitrogen.

The polybutylene terephthalate tweezers used to manipulate the beads did not affect charges on the beads significantly. For example, when we exchanged a charged Nylon or Teflon bead between two pairs of polybutylene terephthalate tweezers 20 times, the charges of the beads increased by only $4 \pm 6 \%$ and $2 \pm 2 \%$ respectively.

Preparation of Charged Beads. In order to charge the beads, we placed nine beads of a desired material (evenly spaced and loosely packed) with an excess (199) of beads of a different material (see the exact arrangement of the beads in Figure 2, top scheme). In our experiments, we charged Nylon (positively-charged) with excess Teflon (negatively-charged), Teflon with excess Nylon, Delrin (positively-charged) with excess Teflon, and Torlon (negatively-charged) with excess Delrin. A linear motor agitated the dish at an amplitude of $9 \mathrm{~mm}$ at $6 \mathrm{~Hz}$ for $3 \mathrm{mins}$ 
under a nitrogen atmosphere (unless other specified), and at a relative humidity of $\sim 1-2 \%$. The beads were free to rotate and collide with each other in the dish.

The Seven-Bead Configuration for Studying Charge Dissipation. We transferred five charged beads from the dish to the bottom substrate (side beads, see Figure 2) and placed a sixth bead (center bead) on top of the five beads. A seventh bead (top bead) was placed on a rigid adhesive surface (double-sided tape on polystyrene).

After rubbing (using the procedure described), the center bead discharged almost completely for all the four polymers studied (Figure 3a). Charge on each side and top bead also decreased the result was an overall reduction in the total charge carried by the seven beads after contact.

Discharge is not specific to this particular experimental setup. When charged beads come into contact with like-charged beads, they discharge; the extent of discharge, however, depends on the fraction of the surface brought into contact. Any other experiment that allows the entire surfaces of the beads to come into contact will result in complete discharge (see SI, Section 3 for another experiment in which like-charged beads discharged when they interacted in a container agitated by a motor). 


\section{a) Same Material}

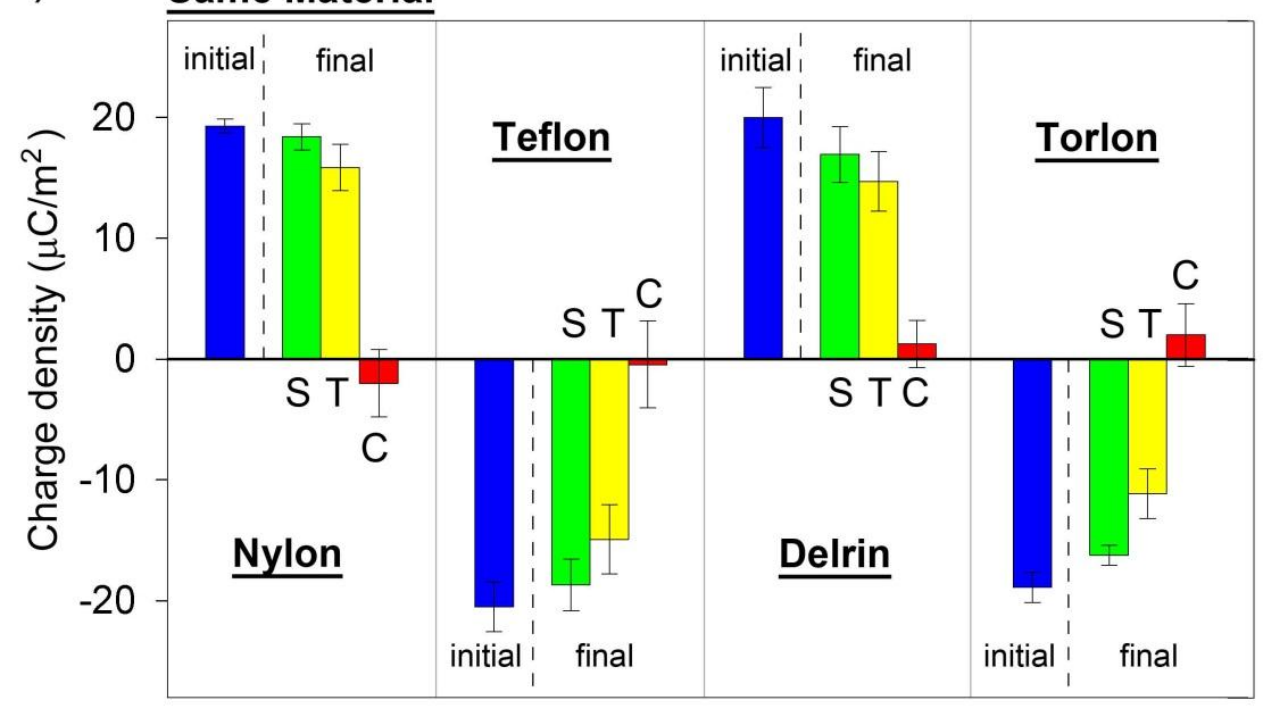

b) Different Materials

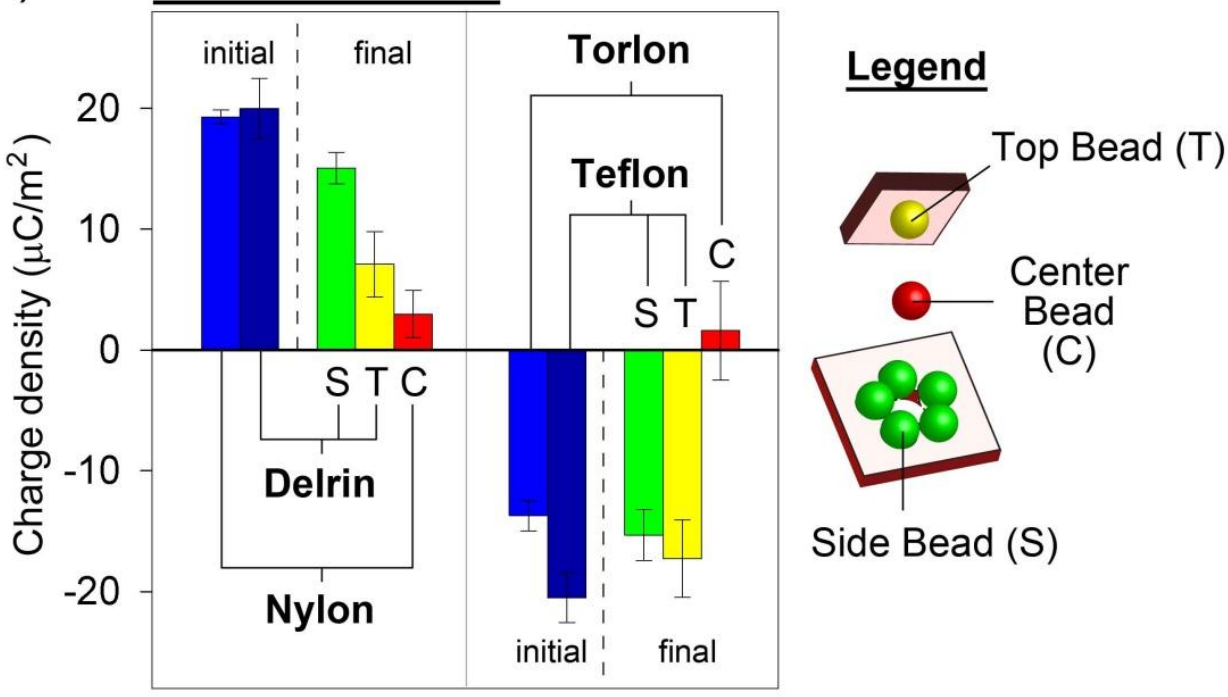

Figure 3. Charge density on the beads before and after contact de-electrification. (a) Bar graphs show the initial (for each bead before contact) and final (after contact) charge densities on the beads when beads of the same materials were brought into contact. These results show that the center bead discharged almost completely for the four types of polymers studied. (b) When beads of different materials were brought into contact, the center bead also discharged. Shown in the graph are two cases in which a mixture of Nylon and Delrin and a mixture of Torlon and Teflon beads were used. ( $n \geq 10$ for all cases) 
Contact de-electrification also occurred for beads of different materials charged to the same polarity. In one experiment, we first charged a mixture of Nylon (positively charged) and Delrin (positively charged) beads against an excess of Teflon (negatively charged) beads. The center bead was Nylon, and the side and top beads were Delrin. In another experiment, we charged a mixture of Teflon (negatively charged) and Torlon (negatively charged) beads against an excess of Nylon (positively charged) beads. In this case, the center bead was Torlon whereas the side and top beads were Teflon. Results (Figure 3b) show that the center beads discharged after contact for both cases.

To summarize the results of these experiments, it seems that insulating polymeric beads regardless of the type of material — that are initially charged to the same polarity, discharge when they come into contact. After the initial charging, charges remained on the beads for hours when left undisturbed. For example, for highly charged Nylon and Teflon beads (approx. \pm 20 $\mu \mathrm{C} / \mathrm{m}^{2}$ ), charges decreased by only $\sim 12 \%$ after 10 hours (in a nitrogen atmosphere with a relative humidity of $1-2 \%)$. So, where did the charges - particularly, the charges on the center bead — go when the beads were brought into contact?

Influence of Atmosphere on Discharge. Contact de-electrification occurred for atmospheres of different dielectric breakdown strengths: helium (0.15), argon (0.5), oxygen (0.87), carbon dioxide (0.88), nitrogen (1), and sulfur hexafluoride (2.5), where the numbers (unitless) in the parenthesis represent the dielectric breakdown strength of the gases relative to nitrogen. ${ }^{24,25}$ Before performing the experiment, we purged the glove bag with one of the gases listed above until the relative humidity dropped to $\sim-3 \%$. Under the same gaseous atmosphere, Nylon beads were charged according to the procedure as illustrated in the top scheme of Figure 2. The charge on the beads after charging increased approximately with increasing dielectric breakdown 
strength of the atmosphere (Figure 4). This trend agrees with previous results from our group ${ }^{18}$ and is compatible with the hypothesis that the amount of charge accumulated on the beads while charging is a result of a balance between contact charging, and discharge due to leakage of charge into the atmosphere (see SI, Section 4 for a discussion on the dependence of the dielectric breakdown strength with the composition of the atmosphere consisting of a mixture of gases).

The charged beads were then discharged according to the same procedure shown in Figure 2. We used the same gaseous atmosphere for both charging and discharging. Despite the differences in initial charge, our results show that the center bead discharges almost completely after contact, in all the different atmospheres investigated. This result suggests that contact deelectrification is general, regardless of the type of gaseous atmosphere involved.

\section{Charge Transfer from Charged Polymeric Beads onto the Solid Substrates is Not}

Significant. One possible mechanism for discharge is that charge (especially on the center bead) dissipated, by some mechanism, into the top and bottom substrates holding the beads. In order to test this possibility, we measured the charges on the substrates and the beads before and after contact de-electrification. The Nylon beads were charged and discharged using the procedure illustrated in Figure 2 under a dry nitrogen atmosphere. The bar graphs in Figure 5 show that there was no significant difference in the magnitude of charge on the solid substrates before and after discharge resulting from bringing the beads into contact; however, there was an overall reduction in total charge of the seven beads. This result shows that charges from the beads did not dissipate into the solid substrates. Results from previous work agree with this finding: there is no detectable migration of charges across polymer surfaces. ${ }^{3,26}$ Thus, it is unlikely that charge migrates/dissipates from the center bead, via other beads, and onto the solid substrates. 


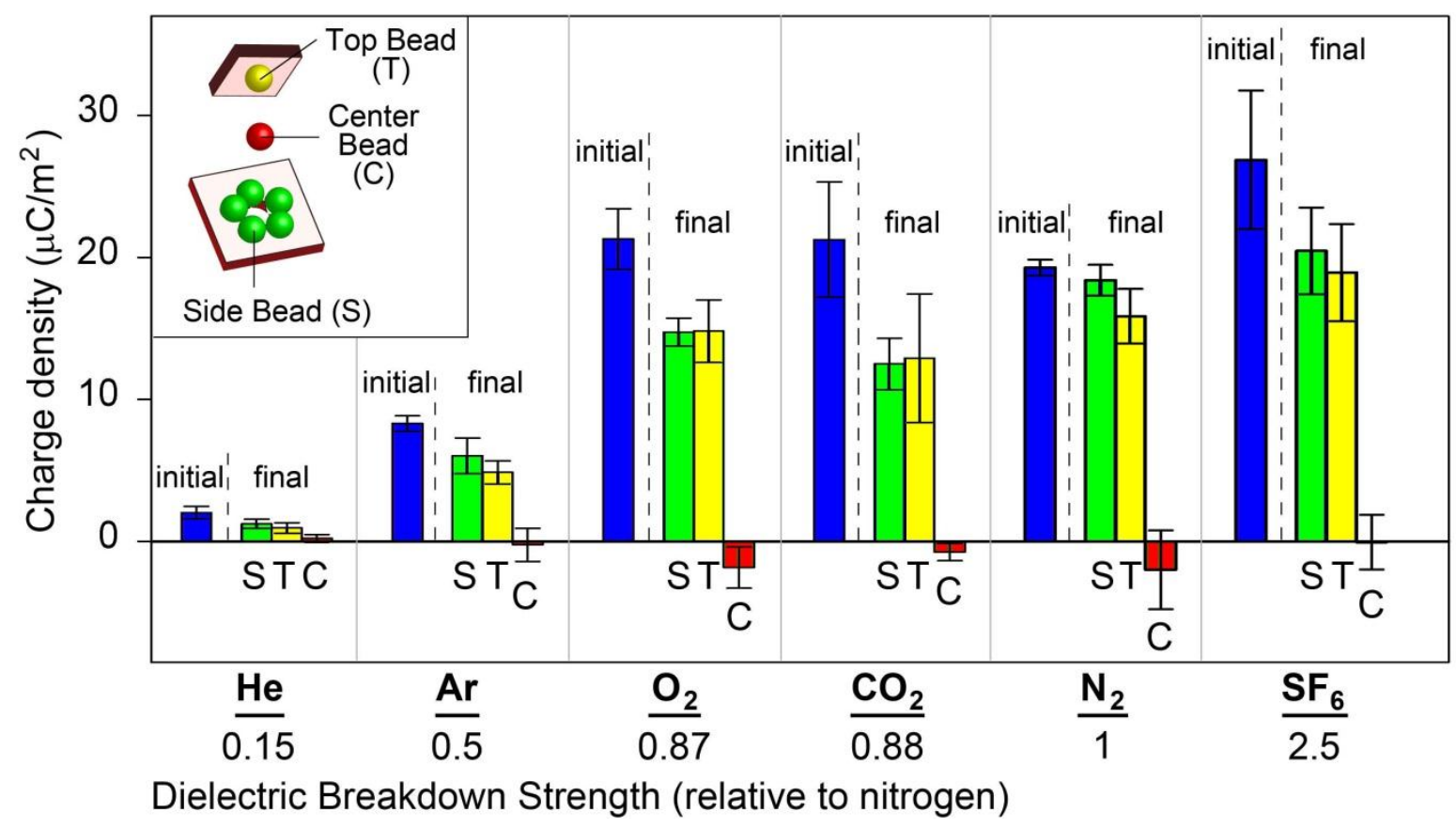

Figure 4. Contact de-electrification occurred under different types of gaseous atmosphere.

Despite different amounts of initial charge on the Nylon beads under different atmospheres, the center bead discharged almost completely after being brought into contact with the top bead for all cases. $(n \geq 7)$ 


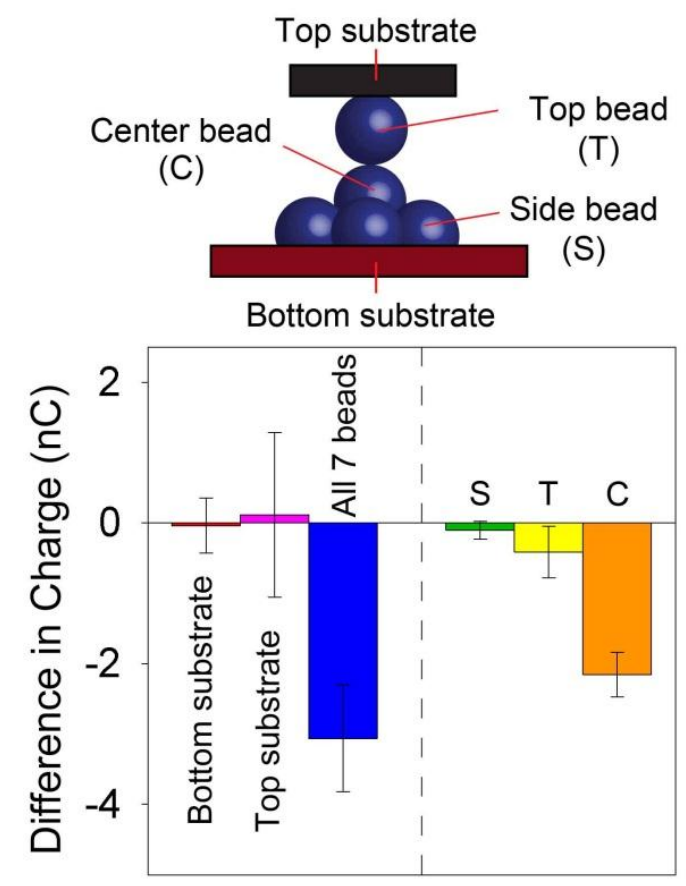

Figure 5. Charge did not dissipate into the solid substrates during contact de-electrification. The charges of the already-charged Nylon beads and the substrates were measured before and after contact de-electrification. Charges on the top and bottom substrates did not change significantly compared to the overall reduction in the charge of all the seven beads involved; this result shows that charges did not transfer from the beads into the substrate. The differences in charge on each side, top and center bead are also shown in this plot. $(n=7)$ 
Charge Dissipates by Transfer to Molecules of Gas in the Surrounding. If charges are not transferred into the surrounding solid substrate, the only mechanism for charge to dissipate is from the beads into the molecules of gas in the atmosphere. Verifying this mechanism requires a direct method of detecting charges transferred from already-charged beads into the atmosphere when the beads come into contact. We hypothesized that the charges transferred to the atmosphere could be measured by a Faraday Cup opened on one side and placed next to interacting charged beads. To demonstrate this hypothesis experimentally, the beads were first charged using the procedure described in Figure 2. Figure 6a shows the experimental setup in which we placed the charged beads on a dish over an aluminum foil and underneath a Faraday Cup with its opened side facing the beads ${ }^{27}$ During agitation, the beads rolled and collided with one another, and contact de-electrification occurred. Charges transferred to the atmosphere as a result of contact de-electrification are collected in the Faraday Cup — we presume by transfer to the inner surface of the Faraday Cup — and led to a reading in the electrometer. More specifically, the sequence of steps in this experiment was as follows (Figure 6b): (1) we grounded the aluminum foil and started recording the charges using the Faraday Cup connected to electrometer; (2) we inserted the dish (PMMA, $3.5 \mathrm{~cm}$ in diameter, neutralized by Zerostat gun) in the space between the aluminum foil and the Faraday Cup and gradually placed 22 already-charged (Nylon or Teflon) beads into the dish; (3) we shook the dish and the beads with a motor (amplitude of $9 \mathrm{~mm}, 4 \mathrm{~Hz}$ ) for 3 mins; (4) finally, we removed the dish and the beads. 
a)

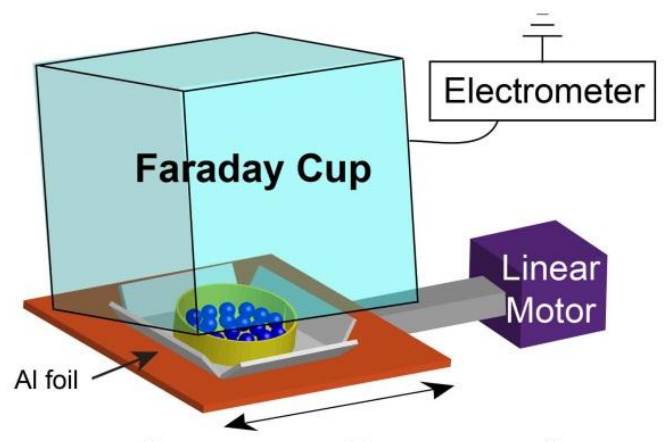

b) 1

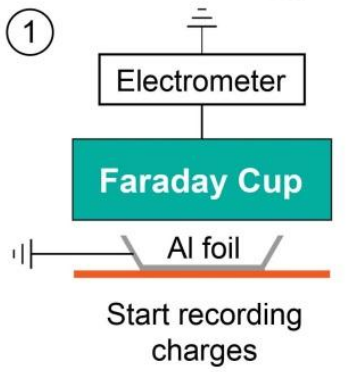

(2)

(3)
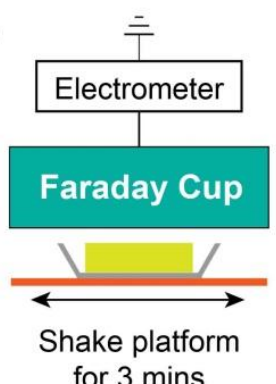

(4)
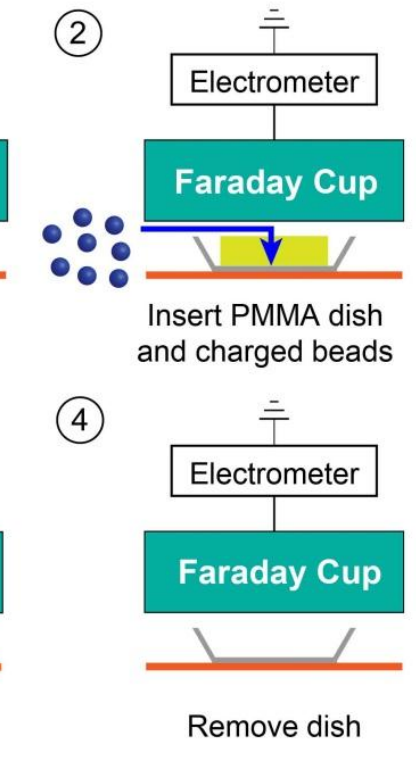

c)

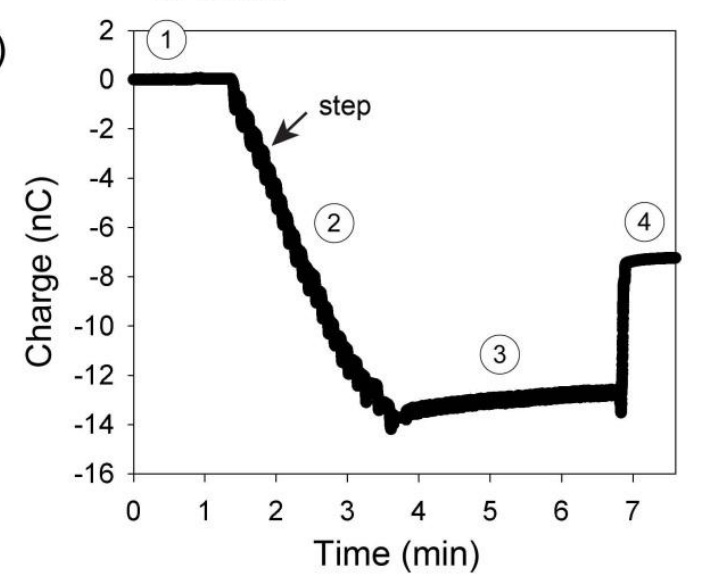

d)

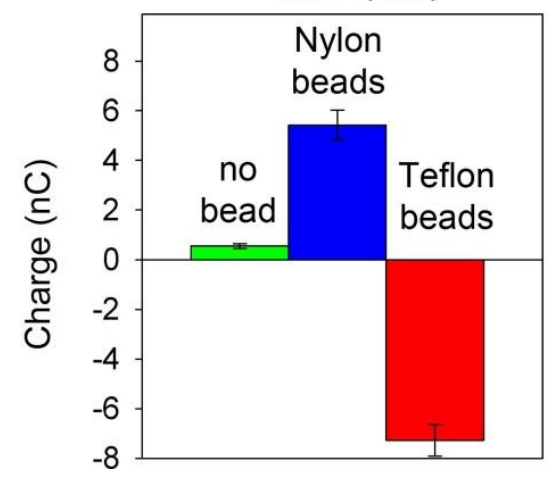


Figure 6. Measurement of charge dissipated into the atmosphere using a Faraday Cup. (a) Scheme shows the Faraday Cup with one of its sides opened and facing a dish filled with charged beads. (b) Illustration showing the four sequential steps involved in the experiment. (c) The plot of the real-time charge measurement (for the case of charged Teflon beads), with numbers in circle representing the respective sequential steps conducted in the experiment described in (b). Each "step" indicated in the plot represents the addition of a bead. (d) Results of measuring differences in charge between step 1 and step 4 for three sets of experiment: with no beads, 22 Nylon beads and 22 Teflon beads in the dish. These results show that charges transferred from the charged beads into the gaseous atmosphere ( $n=7$ for all three experiments). 
Importantly, the beads and dish were not present in both the initial (step 1) and final (step 4) steps. Any difference in charge recorded by the electrometer between steps 1 and 4 thus represented charges dissipated from the beads into the atmosphere. In this experiment, both Nylon and Teflon beads lost $\sim 30 \%$ of their initial charge after agitation; $40 \%$ of the charge out of this $30 \%$ dissipated (or $\sim 10 \%$ of the initial charge on the beads) was detected by the Faraday Cup. This result shows that charge transferred (we presume) from the beads to the gaseous atmosphere, and then to the inner walls of the Faraday Cup (see SI, Section 5 for a discussion on which charge is not dissipated via the PMMA dish to other solid substrates). A control experiment was performed by repeating the procedure without any charged beads in the dish; in this case, no significant charge was measured (Figure 6d).

Theory and Simulation. Equation (1) gives the relationship between the electric field at the surface of the bead, $E_{S}$, and the charge on the bead, $Q$, where $\varepsilon$ is the permittivity and $R$ is the radius of the bead.

$$
E_{S}=\frac{Q}{4 \pi \varepsilon R^{2}}
$$

Since the dielectric breakdown strength of the atmosphere (nitrogen) is $\sim 3.1 \mathrm{MV} / \mathrm{m},{ }^{28}$ the amount of charge storable on the bead at the limit of breakdown is calculated according to Eq. (1) to be $27 \mu \mathrm{C} / \mathrm{m}^{2}$. This charge is close to the initial charge on the beads after charging for all four polymeric materials studied, $\sim \pm 20 \mu \mathrm{C} / \mathrm{m}^{2}$ (see Figure $3 \mathrm{a}$ ). When the charged beads come into contact (Figure 7), the electric field at the region of contact becomes higher and exceeds the dielectric breakdown strength of the atmosphere. In this case, the charges on the surface of the beads will transfer to the gas molecules in the atmosphere. 
We simulated the electric field surrounding a bead and two beads in contact (Figure 7b-c) assuming a constant surface charge density of $20 \mu \mathrm{C} / \mathrm{m}^{2}$ on the bead using Finite Element Method (Comsol Multiphysics 4.1). The simulation of a single bead shows that the maximum electric field surrounding a single bead is lower than the dielectric breakdown strength of the atmosphere $(3.1 \mathrm{MV} / \mathrm{m})$. The simulation of two beads in contact, however, shows a region in which the electric field exceeded 3.1 MV/m around the area of contact as marked by the region within the black dashed line (Figure 7c).

When the electric field surrounding the beads exceeds the dielectric breakdown strength of the atmosphere, gas molecules ionize and generate ions (in principle, both positive and negative) and electrons. ${ }^{29}$ Depending on the electrical polarity of the surface of the beads, one of the two species, i.e., positive ions or the electrons, generated from the ionization of gaseous molecules are attracted to the surface while the other species are repelled from the surface. The charged species attracted to, and subsequently, deposited on the surface of the beads causes the beads to discharge.

The liberation of an electron from a neutral gas molecule and the acceleration of the electron due to the electric field of the beads can result in multiple collisions between the electron and other gas molecules. These collisions can produce pairs of positive ions and electrons, which can accelerate and collide with other gas molecules, and thus produce more charged species. This cascade of collisions multiplies the number of charged species and could, hypothetically, generate enough charged species to discharge the beads completely (as observed in our experiments). For a corona discharge from a hemispherical electrode (diameter $1 \mathrm{~mm}$ ) maintained at $-15 \mathrm{kV}$, the current density ${ }^{30}$ measured on a plane electrode placed at $4 \mathrm{~cm}$ away is 
$\sim 0.5 \mu \mathrm{A} / \mathrm{cm}^{2}$; this current can discharge a bead of $1 / 4$ inch in diameter $\left(\sim 10^{10}\right.$ charges/bead) used in our experiments in $\sim 4 \mathrm{~ms}$.

Controlling Charge by Contact de-Electrification. Contact de-electrification could be used to control the amount of charge on a solid surface. One advantage of using contact deelectrification to control the amount of charge is that the discharge process is rapid — discharge completes in a single contact (as shown by the experiment described in the next paragraph). In contrast, contact electrification typically requires multiple contacts before the materials charge up to their steady-state values.

We demonstrated rapid discharge by bringing two charged slabs of Nylon or Teflon $(3 \mathrm{~cm} \times$ $3 \mathrm{~cm} \times 1.5 \mathrm{~mm}$ each) into contact. The Nylon (or Teflon) slabs were first charged by rubbing against another piece of Teflon (or Nylon). After charging the slabs, the two like-charged slabs were brought into contact manually (by pushing the corners and the center of both slabs together for $\sim 3 \mathrm{sec}$ to ensure that the entire surfaces of the slabs were contacted), for five times. Charge measurements using the Faraday Cup (Figure 8) showed that the slabs discharged on the first contact; subsequent (five) contacts did not alter the charge on the two slabs significantly. A single contact was thus sufficient to discharge the slabs.

For both Nylon and Teflon slabs, some charges remained on the slabs after contact (Figure 8). This remaining charge, however, could be due to effects unrelated to contact deelectrification. In a control experiment, Nylon or Teflon slabs initially neutralized by spraying ions from an antistatic Zerostat gun were brought into contact the same way as the charged slabs. Results show that the slabs became charged; these charges could thus originate from tribocharging of the materials when the slabs were manipulated manually. 
a)

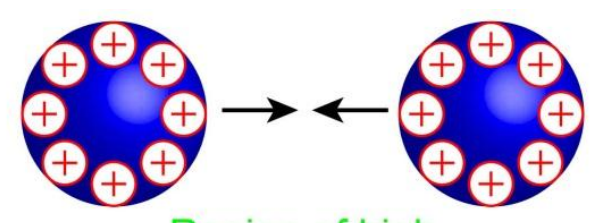

Region of high

electric field

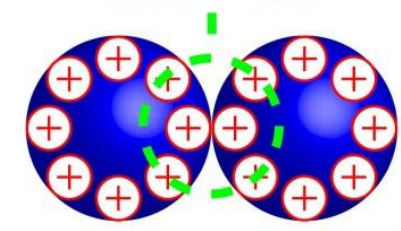

Charges transferred into

gaseous atmosphere
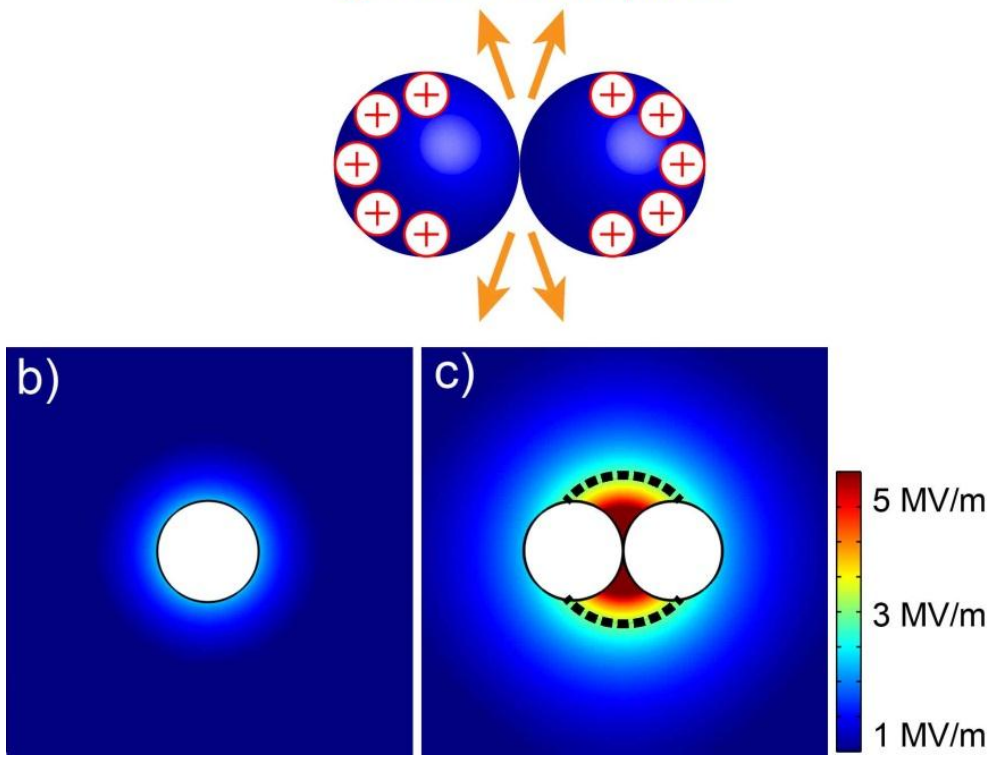

Figure 7. Proposed mechanism for contact de-electrification. (a) When charged beads come into contact, the magnitude of the electric field, $E$, increases at the point of contact. If $E$ exceeds the dielectric breakdown strength of the surrounding gaseous atmosphere, charges will dissipate into the atmosphere, and neutralize the bead at the region of contact. (b-c) Images show the numerical simulations of the electric field surrounding (b) one bead and (c) two beads in contact where each bead has a constant surface charge density of $20 \mu \mathrm{C} / \mathrm{m}^{2}$ (typical charge of the beads after charging by the procedure described in Figure 2). The black dashed lines in (c) indicate the iso- 
contour lines at which the electric field is $3.1 \mathrm{MV} / \mathrm{m}$, the dielectric breakdown strength of nitrogen (atmosphere used in the experiments). The area within the dashed lines shows the region where the electric field exceeds the dielectric breakdown strength of nitrogen - that is, the region where charges could be transferred into the atmosphere. 


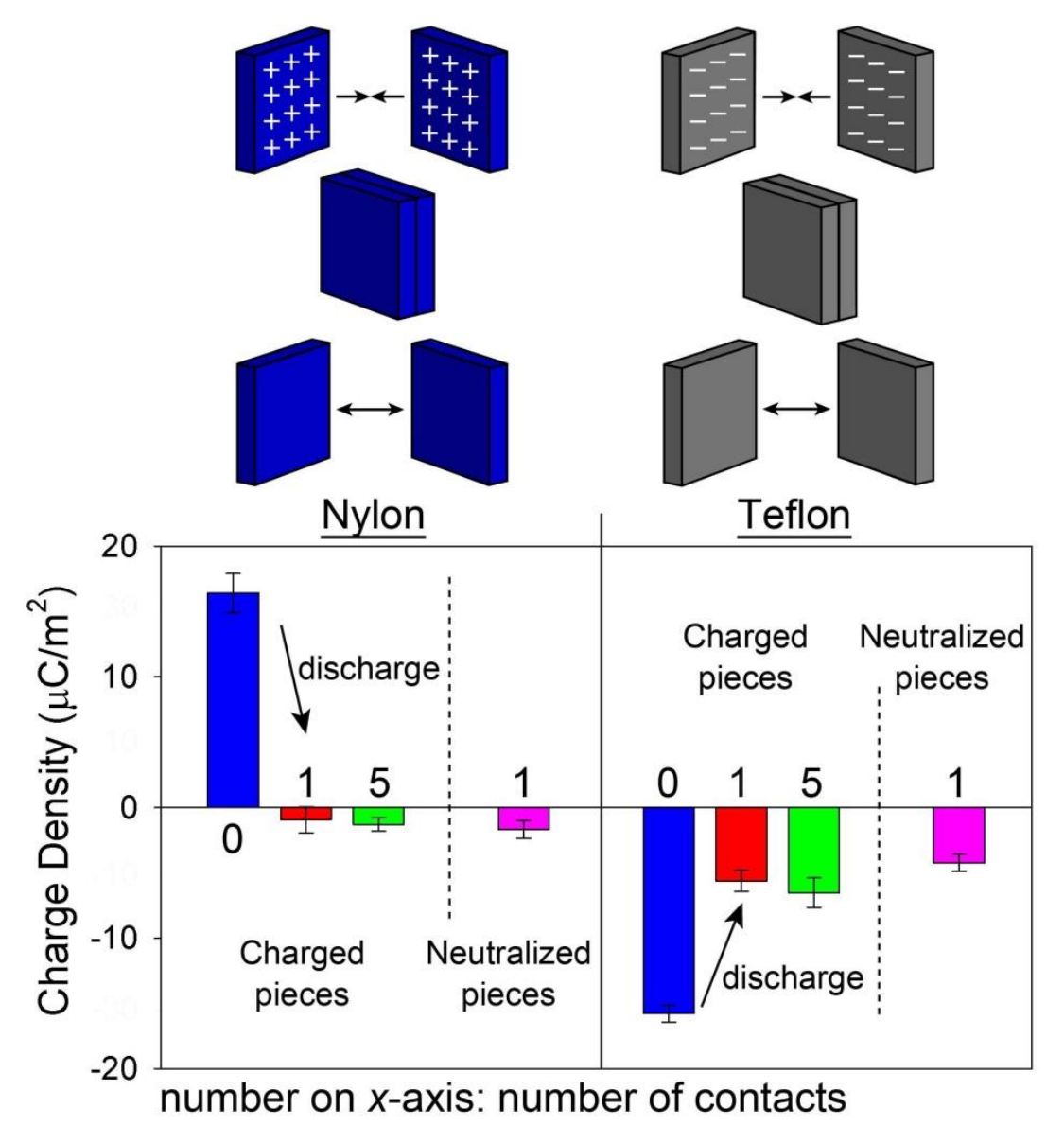

Figure 8. Contact de-electrification is completed in a single contact. When two slabs of likecharged ("Charged pieces") Nylon or Teflon came into contact, they discharged on the first contact. Subsequent contacts (five) showed no further discharge. The charges remaining on the slabs are probably due to tribocharging of the slabs during contact: control experiments were conducted on initially neutralized slabs ("Neutralized pieces") by Zerostat gun. These slabs became slightly charged on contact. ( $n=7$ for all cases) 


\section{CONCLUSION}

This study demonstrates that the contact of like-charged polymeric beads can result in loss of charge; a phenomenon which we refer to as contact de-electrification. Known examples of discharge in insulating solids as a result of dielectric breakdown include field ionization, partial discharge and the formation of Lichtenberg figures (or electrical "treeing"), which typically take place when the solids are placed under a high, external voltage. ${ }^{29}$ To our knowledge, this study presents the first evidence of electrical discharge in like-charged polymers — charged simply by contact electrification - when they are brought into contact.

We infer that this discharge involves transfer of charge from the charged beads into the molecules of gas in the atmosphere when the electric field at the point of contact exceeds the dielectric breakdown strength of the gaseous atmosphere. Importantly, this mechanism implies that contact de-electrification should be general and perhaps, even universal, to electrostatically charged insulators - that is, contact de-electrification should occur under any type of gaseous atmosphere and with any type of material, as long as the electric field at the point of contact between the materials exceeds the dielectric breakdown strength of the atmosphere. This implication is compatible with our experiments, in which contact de-electrification occurred for different types of materials, and under different atmospheric conditions. We expect the same reasoning to apply to non-polymeric insulators charged to the same polarity.

In order to establish a complete understanding of contact electrification among interacting particles, it is desirable to construct a theoretical framework that involves all the relevant mechanisms, including electron transfer, ion transfer, material transfer and contact deelectrification. An analogy of this theoretical framework is the so-called "population balance 
model", used for modeling processes such as crystallization and precipitation; ${ }^{31}$ this model consists of a partial differential equation which includes sub-processes such as nucleation, growth, dissolution, aggregation and breakage. By taking into account of all the different mechanisms that govern nucleation, it is possible to simulate and predict the size distribution of crystals resulting from different types of flows, with application for a number of large scale

processes. ${ }^{32} \mathrm{We}$ envision the same process could be applied to the flow of granular particles: through modeling the different processes involved (empirically or semi-empirically), it will be possible to predict the spatial and temporal distribution of charges of the granular particles in flowing processes.

\section{ACKNOWLEDGMENTS}

This work was supported by a subcontract from Northwestern University from the Department of Energy (DE-SC0000989).

\section{ASSOCIATED CONTENT}

A detailed description of the experimental procedures, an experiment on undyed Nylon beads, an alternative experimental setup for contact de-electrification, a brief description of the dependence of dielectric breakdown strength with composition of the gaseous atmosphere, and a discussion that charge did not dissipate from the beads into other solid substrates. This material is available free of charge via the Internet at http://pubs.acs.org. 


\section{REFERENCES:}

(1) McCarty, L. S.; Whitesides, G. M. Angew. Chem.-Int. Edit. 2008, 47, 2188-2207.

(2) Williams, M. W. AIP Adv. 2012, 2, 010701.

(3) Baytekin, H. T.; Patashinski, A. Z.; Branicki, M.; Baytekin, B.; Soh, S.; Grzybowski, B.

A. Science 2011, 333, 308-312.

(4) Lacks, D. J.; Sankaran, R. M. J. Phys. D: Appl. Phys. 2011, 44, 453001.

(5) Harper, W. R. Contact and Frictional Electrification; Oxford University Press: London, 1967.

(6) Zink, J. I. Accounts Chem. Res. 1978, 11, 289-295.

(7) Diaz, A. F.; Felix-Navarro, R. M. J. Electrost. 2004, 62, 277-290.

(8) Baytekin, H. T.; Baytekin, B.; Incorvati, J. T.; Grzybowski, B. A. Angew. Chem.-Int. Edit. 2012, 51, 4843-4847.

(9) Shaw, P. E.; Hanstock, R. F. Proc. R. Soc. Lond. Ser. A-Contain. Pap. Math. Phys. Character 1930, 128, 474-480.

(10) Apodaca, M. M.; Wesson, P. J.; Bishop, K. J. M.; Ratner, M. A.; Grzybowski, B. A. Angew. Chem.-Int. Edit. 2010, 49, 946-949.

(11) Lowell, J.; Roseinnes, A. C. Adv. Phys. 1980, 29, 947-1023.

(12) Akande, A. R.; Lowell, J. J. Phys. D-Appl. Phys. 1987, 20, 565-578.

(13) Liu, C. Y.; Bard, A. J. J. Am. Chem. Soc. 2009, 131, 6397-6401.

(14) Piperno, S.; Cohen, H.; Bendikov, T.; Lahav, M.; Lubomirsky, I. Angew. Chem.-Int. Edit. 2011, 50, 5653-5656.

(15) Baytekin, B.; Baytekin, H. T.; Grzybowski, B. A. J. Am. Chem. Soc. 2012, 134, 72237226. 
(16) Piperno, S.; Cohen, H.; Bendikov, T.; Lahav, M.; Lubomirsky, I. Phys. Chem. Chem. Phys. 2012, 14, 5551-5557.

(17) McCarty, L. S.; Winkleman, A.; Whitesides, G. M. Angew. Chem.-Int. Edit. 2007, 46, 206-209.

(18) McCarty, L. S.; Winkleman, A.; Whitesides, G. M. J. Am. Chem. Soc. 2007, 129, 40754088.

(19) Diaz, A. F.; Fenzel-Alexander, D. Langmuir 1993, 9, 1009-1015.

(20) Thomas, S. W.; Vella, S. L.; Kaufman, G. K.; Whitesides, G. M. Angew. Chem.-Int. Edit. 2008, 47, 6654-6656.

(21) Vella, S. J.; Chen, X.; Thomas, S. W.; Zhao, X. H.; Suo, Z. G.; Whitesides, G. M. J. Phys. Chem. C 2010, 114, 20885-20895.

(22) Wiles, J. A.; Grzybowski, B. A.; Winkleman, A.; Whitesides, G. M. Anal. Chem. 2003, $75,4859-4867$.

(23) Cademartiri, R.; Stan, C. A.; Tran, V. M.; Wu, E.; Friar, L.; Vulis, D.; Clark, L. W.; Tricard, S.; Whitesides, G. M. Soft Matter 2012, DOI: 10.1039/c1032sm26192h.

(24) Vijh, A. K. IEEE T. Electr. Insul. 1982, 17, 84-87.

(25) Brand, K. P. IEEE T. Electr. Insul. 1982, 17, 451-456.

(26) Chudleigh, P. W. J. Appl. Phys. 1977, 48, 4591-4596.

(27) The Faraday Cup is very sensitive to vibration; therefore, we kept the Faraday Cup stationary, and not connected to the moving platform (particularly, not connected to the aluminum foil).

(28) CRC Handbook of Chemistry and Physics; 84th ed.; Lide, D. R., Ed.; CRC Press, 2004. 
(29) Naidu, M. S.; Kamaraju, V. High Voltage Engineering; 4th ed.; McGraw-Hill: New Delhi, 2009.

(30) Tassicker, O. J. Electron. Lett. 1969, 5, 285-286.

(31) Marchal, P.; David, R.; Klein, J. P.; Villermaux, J. Chem. Eng. Sci. 1988, 43, 59-67.

(32) Woo, X. Y.; Tan, R. B. H.; Chow, P. S.; Braatz, R. D. Cryst. Growth Des. 2006, 6, 12911303. 
TOC:

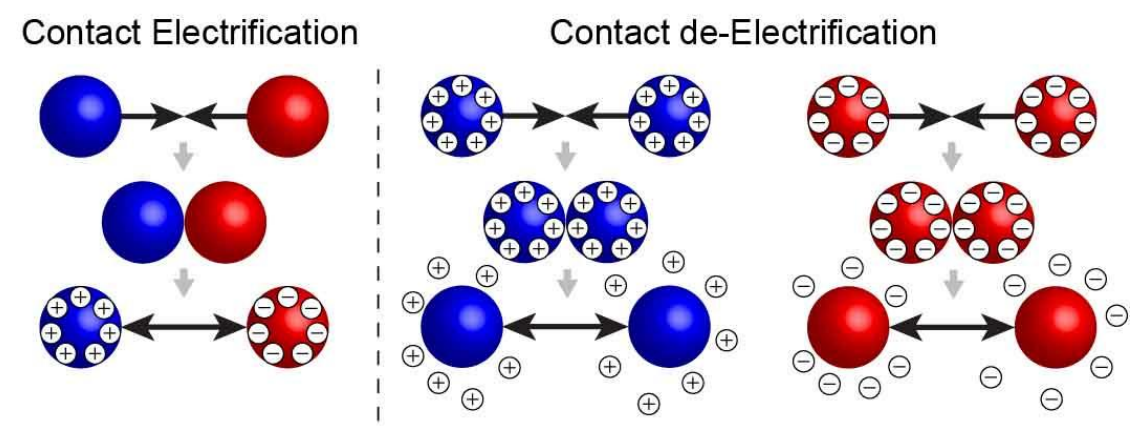

